\section{To peel or not to peel the internal limiting membrane: a question finally answered?}

\author{
David Wong
}

For the surgical treatment of idiopathic thickness macular hole, the question of whether to peel or not to peel the internal limiting membrane (ILM) has been asked repeatedly since at least $2002 .{ }^{12}$ Most surgeons carry out ILM peeling routinely. Many are already convinced of its benefit. Is it worthwhile conducting a randomised trial when the clinical practice of ILM peel has already been widely adopted? Is the answer simply academic?

If all macular holes respond in the same way to surgery, the answer might indeed be academic. They do not. It is now clear that smaller holes close more readily. ${ }^{3} \mathrm{~A}$ lesser intervention may be appropriate for the treatment of smaller holes. For example, a recent randomised trial comparing a facedown versus a seated position found that the success rate in idiopathic macular holes smaller than $400 \mu \mathrm{m}$ was not influenced by postoperative posture. ${ }^{4}$

On the other hand, if the maximum intervention works best, why not apply it to every patient? After all, there is a price to pay for failure in primary closure. Holes that do not close after the first vitrectomy tend to increase in size, and even when they are closed with a repeat operation, the visual results are often poor. ${ }^{5}$ The point is that the maximum algorithm has its drawbacks. The use of long-acting gases and head-down posture may simply be inconvenient for patients. ILM peeling and preretinal manipulations may importantly be associated with retinal pigment epithelial changes, ${ }^{6}$ trauma to the neurosensory retina, phototoxicity resulting from prolonged manipulations and possible adverse reactions to the dyes used.

In this issue (see page 1005), Christensen carried out a randomised controlled trial of no peel versus peel using Indocyanine Green (ICG) in a single centre, Glostrup Hospital in Denmark. ${ }^{8}$ This wellconducted study aimed at examining the value of ILM peeling in stage- 2 and -3 idiopathic macular hole surgery. The

Correspondence to: Dr David Wong, St Paul's Eye Unit, Royal Liverpool University Hospital, Prescot Street,

Liverpool L7 8XP, UK; shdwong@liv.ac.uk results show that surgery with ILM peeling, for both stage 2 and -3 macular hole, is associated with a significantly higher closure rate than surgery without ILM peeling (stage 2: $100 \%$ vs $55 \%$; stage 3: $90 \%$ vs $36 \%)$. The results seem convincing.

Does this mean that the question is now finally answered? Yes and no. There may still be room for doubt, given that randomisation was changed after 40 patients were recruited. The interim analysis of stage 3 holes showed that the peeled group did significantly better. The stopping rule for randomisation meant that it was unethical to continue. For the remainder of the trial, the patients with stage 3 holes were randomised 2:1 to ILM peeling with Trypan Blue (TB) or ICG. The randomisation to no peeling stopped after 14 stage 3 holes were recruited (presumably because nine holes failed to, as the anatomical success rate in this group was $36 \%$ ). Is this a representative result, or is it a statistical quirk due to clustering of failures? Brooks, in a nonrandomised study, achieved with no ILM peeling an anatomical success rate of $82 \%$; 9 Margherio et al, in a consecutive study, achieved with no ILM peeling an anatomical closure rate of $86 \% .{ }^{10}$

The majority of the patients $(>80 \%)$ in this Danish trial were rendered pseudophakic 4 weeks prior to the vitrectomy. This was done in order to eliminate one of the major confounding factors that dogged many previous studies on macular hole surgery, namely progressive lens opacity following vitrectomy affecting the visual outcome. However, could the cataract surgery have contributed to the unusually low anatomical success rate of non-peeling group? A recent study on patients who had cataract surgery before, after or combined with vitrectomy and vitrectomy alone for idiopathic macular hole found that cystoid macular oedema was associated with a sevenfold increase in reopening of the closed macular holes. ${ }^{11}$ There is a high incidence of cystoid macular oedema following cataract surgery, the majority being subclinical. ${ }^{12}$ Equally, ILM peeling is a recognised treatment for cystoid macular oedema in conditions such as diabetic retinopathy and retinal vein occlusion. ${ }^{13}{ }^{14}$ Is it conceivable that by rendering the patients pseudophakic prior to the vitrectomy, the trial favoured ILM peeling and disadvantaged the no-peeling group?

What started off as a study to address primarily a single research question, namely, "Is peeling better than no peeling?", effectively became a three-arm trial of no dye (and no peel) versus ICGassisted peeling versus TB-assisted peeling. Nonetheless, the information yielded by the second randomisation is both interesting and relevant. The study concluded that "intraoperative ILM staining with $0.05 \%$ isotonic ICG was not associated with a significantly different visual outcome than non-peeling or TB peeling in eyes with primary hole closure." One of the many strengths of this study was the recording of postoperative visual fields in all patients. A visual-field defect occurred in one of 35 patients treated with ICG, one of 18 patients with TB and none with non-peeling. Those surgeons who use ICG routinely might take comfort from the results of this trial and conclude that ICG at a concentration of $0.05 \%$ is safe. Equally, those who have experienced unexplained visual-field loss following ICG-assisted peel might believe that the adverse reaction to this dye is idiosyncratic and as such not likely to be detected in a relatively small number of patients.

There is still variation in our approaches to the treatment of idiopathic macular hole. There are those surgeons who do no peeling, those who peel but use no dye, those who use ICG-assisted peel and yet others who are switching to the newer and purported "safer" dyes. ${ }^{15}$ It is fair to say that the majority are already persuaded by the efficacy of ILM peeling, and Christensen et al through their meticulous study will convince even more surgeons to peel routinely. It is noteworthy that the only other randomised trial of vitrectomy with or without ILM peeling of for idiopathic macular hole in Chinese patients found a very similar anatomical closure rate of $92 \%$ and $32 \%$ respectively. ${ }^{16}$ There is a UK multicentre randomised trial of ILM peel versus no peel that has finished recruiting. ${ }^{17}$ (FILMS is registered with Current Controlled Trials ISRCTN number 33175422 and Clinical Trials.gov identifier NCT00286507.) It would be interesting to see if the British results corroborated that of the Danish trial.

Competing interests: None. 
Br J Ophthalmol 2009;93:987-988.

doi:10.1136/bjo.2008.156521

\section{REFERENCES}

1. Kuhn F. Point: to peel or not to peel, that is the question. Ophthalmology 2002;109:9-11.

2. Hassan T, Williams GA. Counterpoint: to peel or not to peel: is that the question? Ophthalmology 2002; 109:11-12.

3. Tadayoni R, Gaudric A, Haouchine B, et al. Relationship between macular hole size and the potential benefit of internal limiting membrane peeling. Br J Ophthalmol 2006;90:1239-41.

4. Guillaubey A, Malvitte L, Lafontaine PO, et al. Comparison of face-down and seated position after idiopathic macular hole surgery: a randomized clinical trial. Am J Ophthalmol 2008;146:128-34.

5. Valldeperas $\mathbf{X}$, Wong $\mathrm{D}$. Is it worth reoperating on macular holes? Ophthalmology 2008;115:158-63.

6. Poliner LS, Tornambe PE. Retinal pigment epitheliopathy after macular hole surgery. Ophthalmology 1992;99:1671-7.
7. Engelbrecht NE, Freeman J, Sternberg P Jr, et al. Retinal pigment epithelial changes after macular hole surgery with indocyanine green-assisted internal limiting membrane peeling. Am J Ophthalmol 2002; 133:89-94.

8. Christensen UC, Krøyer K, Sander B, et al. Value of internal limiting membrane peeling in surgery for idiopathic macular hole stage 2 and 3-a randomized clinical trial. Br J Ophthalmol 2009;93:1005-1015.

9. Brooks HL Jr. Macular hole surgery with and without internal limiting membrane peeling. Ophthalmology 2000;107:1939-48.

10. Margherio RR, Margherio AR, Williams GA, et al. Effect of perifoveal tissue dissection in the management of acute idiopathic full-thickness macular holes. Arch Ophthalmol 2000;118:495-8.

11. Bhatnagar $\mathbf{P}$, Kaiser PK, Smith SD, et al. Reopening of previously closed macular holes after cataract extraction. Am J Ophthalmol 2007;144:252-9.

12. Ching HY, Wong AC, Wong CC, et al. Cystoid macular oedema and changes in retinal thickness after phacoemulsification with optical coherence tomography. Eye 2006;20:297-303.
13. Patel JI, Hykin PG, Schadt M, et al. Pars plana vitrectomy with and without peeling of the inner limiting membrane for diabetic macular edema. Retina 2006;26:5-13

14. Mandelcorn MS, Nrusimhadevara RK. Internal limiting membrane peeling for decompression of macular edema in retinal vein occlusion: a report of 14 cases. Retina 2004;24:348-55.

15. Remy $\mathbf{M}$, Thaler S, Schumann RG, et al. An in vivo evaluation of Brilliant Blue $\mathrm{G}$ in animals and humans. Br J Ophthalmol 2008;92:1142-7.

16. Kwok AK, Lai TY, Wong VW. Idiopathic macular hole surgery in Chinese patients: a randomised study to compare indocyanine green-assisted internal limiting membrane peeling with no internal limiting membrane peeling. Hong Kong Med J 2005;11:259-66.

17. Lois N, Burr J, Norrie J, et al. Clinical and costeffectiveness of internal limiting membrane peeling for patients with idiopathic full thickness macular hole. Protocol for a Randomised Controlled Trial: FILMS (Full-thickness macular hole and Internal Limiting Membrane peeling Study). Trials 2008;3:61. 